\title{
Redshift of a photon emitted along the black hole horizon
}

\author{
A. V. Toporensky ${ }^{1,2, a}$, O. B. Zaslavskii ${ }^{2,3, b}$ \\ ${ }^{1}$ Sternberg Astronomical Institute, Lomonosov Moscow State University, Moscow, Russia \\ 2 Kazan Federal University, Kremlevskaya 18, Kazan 420008, Russia \\ ${ }^{3}$ Department of Physics and Technology, Kharkov V.N. Karazin National University, 4 Svoboda Square, Kharkov 61022, Ukraine
}

Received: 30 December 2016 / Accepted: 8 March 2017 / Published online: 21 March 2017

(C) The Author(s) 2017. This article is an open access publication

\begin{abstract}
In this work we derive some general features of the redshift measured by radially moving observers in the black hole background. Let observer 1 cross the black hole horizon emitting a photon, while observer 2 crossing the same horizon later receives it. We show that if (i) the horizon is the outer one (event horizon) and (ii) it is nonextremal, the received frequency is redshifted. This generalizes recent results in the literature. For the inner horizon (like in the Reissner-Nordström metric) the frequency is blueshifted. If the horizon is extremal, the frequency does not change. We derive explicit formulas describing the frequency shift in generalized Kruskal- and Lemaitre-like coordinates.
\end{abstract}

\section{Introduction}

Redshift is one of the well-known effects of gravity; it plays an essential role in relativistic astrophysics. Its description entered many textbooks. In particles, it concerns propagation of light in the black hole background. Thus, much attention is focused on the properties of light outside the event horizon. Less these properties inside the horizon are discussed. Also, strange as it may seem, the question of the redshift of a photon moving along the horizon dropped out from consideration almost completely. There is general discussion of this issue in [1] for the Reissner-Nordström-de Sitter metric but the relation between redshift or blueshift and the nature of the horizon was not revealed there.

Meanwhile, there are several points that can serve as a motivation for such a consideration.

(i) Recently, important methodic issues were discussed in $[2,3]$ concerning properties of the world visible by an observer falling into the Schwarzschild black hole and

\footnotetext{
a e-mail: atopor@rambler.ru

b e-mail: zaslav@ukr.net
}

communicating by radio signals with another falling one. In doing so, some incorrect statements were made in [2] about "ghosts" of the first observer supposedly waiting for the second one on the horizon. As was argued in [3], there are no such ghosts at all, although the second observer does receive a signal from the first one emitted at the moment of crossing the horizon. This required detailed calculation of the frequency shift for a photon propagating along the horizon with the result that a finite redshift occurs in this case.

In the present work, we generalize these observations, demonstrate that such a redshift is present for any horizon of a spherically symmetric nonextremal black hole and find its value.

(ii) If the metric contains an inner horizon (say, for the Reissner-Nordström black hole), the calculation of the frequency shift for a photon emitted along such a horizon is of special interest. We demonstrate that now, instead of a redshift, a blueshift occurs. In the limit when a photon is received near the bifurcation point, the blueshift becomes infinite. This establishes the connection of the issue under discussion with the analog of the BañadosSilk-West (BSW) effect [4]. It consists in the infinite growth of the energy of colliding particles in the center of mass frame. Originally, it was found near the event horizon but, later on, it turned out that another similar effect is valid also near the inner horizon (see [5] and the references therein). On the other hand, the issue under discussion can be considered as an effect supplemental to a well-known instability of the inner horizon $[6,7]$.

(iii) In addition to the propagation along the nonextremal horizon, there is also the question of what happens in the extremal case. We argue that, by contrast to the two previous ones, now the frequency shift is absent.

(iv) In Refs. [2,3] the Kruskal-Szekerez (KS) coordinate system was used. We also exploit it. In addition, it is of interest to compare the results using another pow- 
erful system - the Lemaitre one. We construct such a system for a whole class of metrics that includes the Schwarzchild one as a particular case.

In the present work, we restrict ourselves to the simplest case of radially moving observers.

\section{Motion outside the nonextremal event horizon}

We consider the metric

$\mathrm{d} s^{2}=-f \mathrm{~d} t^{2}+\frac{\mathrm{d} r^{2}}{f}+r^{2}\left(\mathrm{~d} \theta^{2}+\sin ^{2} \theta \mathrm{d} \phi^{2}\right)$.

We suppose that the metric has the event horizon at $r=r_{+}$, so $f\left(r_{+}\right)=0$. (For simplicity, we assumed that $g_{00} g_{11}=-1$ but this condition can be relaxed easily.) We consider now a nonextremal black hole. Near the horizon,

$f \approx \kappa\left(r-r_{+}\right)$

where $\kappa=\frac{f^{\prime}\left(r_{+}\right)}{2}$ is the surface gravity.

Let an observer have the four-velocity $u^{\mu}=\frac{\mathrm{d} x^{\mu}}{\mathrm{d} \tau}$, where $\tau$ is the proper time. We restrict ourselves to the radial motion of a massive particle (we call it "observer"). Then the fourvelocity $u^{\mu}=(\dot{t}, \dot{r})$. Here, a dot denotes the derivative with respect to $\tau$.

The geodesic equations of motion for such a particle read

$m \dot{t}=\frac{E}{f}$,

$m \dot{r}=-Z, Z=\sqrt{E^{2}-f m^{2}}$,

$E=-m u_{t}$ is the conserved energy of a particle.

For a photon having the wave vector $k^{\mu}$, the equations of motion are

$k^{t}=\frac{\omega_{0}}{f}$,

$k^{\phi}=\frac{l}{g_{\phi}}$,

$k^{r}=-Q, Q=\sqrt{\omega_{0}^{2}-f \frac{l^{2}}{r^{2}}}$,

where $\omega_{0}=-k_{0}$ is the conserved frequency, $l=k_{\phi}$ is the conserved angular momentum.

Let a free falling observer emit or receive a photon. Its frequency measured by this observer is equal to

$\omega=-k_{\mu} u^{\mu}$.

Taking into account (3)-(7), we find after straightforward calculation:

$\omega=\frac{E \omega_{0}-\varepsilon Z Q}{m f}$.
Here, $\varepsilon=+1$ if both objects (the observer and the photon) move in the same direction and $\varepsilon=-1$ if they do this in opposite ones. If $\varepsilon=-1$, this corresponds to a head-on collision between a massive and a massless particles and this means that either an observer receives a photon emitted from a smaller value of $r$ or a falling observer emits a photon in the backward direction.

Near the horizon, $f \ll 1$. Let $\varepsilon=+1$ and the observer looks back. Near the horizon, he will see the frequency

$\omega \approx \frac{1}{2}\left(\frac{E l^{2}}{m \omega_{0} r_{+}^{2}}+\frac{\omega_{0} m}{E}\right)$.

If $\varepsilon=-1$,

$\frac{\omega}{\omega_{0}} \approx \frac{2 E}{m f_{\mathrm{em}}}$.

Here $f_{\mathrm{em}}$ is the value of the metric function in the point of emission. Let the observer emit in his frame a photon having the frequency $\omega$, traveling to infinity. Then at infinity it will be received with the frequency $\omega_{0}$ having the order $f_{\text {em }}$. Equation (11) agrees with the standard result for the Schwarzschild metric (see Sect. XII.102 of [8], especially Eq. 102.10).

It is instructive to compare this with another situation usually discussed in textbooks when the emitter is not free falling but is static. In the latter case, the frequency at infinity $\omega_{0}=\omega \sqrt{f}$ (see, e.g. Eq. 88.6 of [8]) has the order $\sqrt{f_{\mathrm{em}}}$. Obviously, the difference can be attributed to the motion of an emitter (the Doppler effect makes the redshift stronger).

Some reservations are in order. Throughout the paper, we assume that the geometric optics is a reasonable approximation for propagation of light waves. As usual, this implies that the wavelength $\lambda$ satisfies the relations $\frac{\lambda}{2 \pi} \ll \mathcal{L}, \mathcal{R}$, where $\lambda$ is the wave length, $\mathcal{L}$ is a typical scale characterizing a wave packet and $\mathcal{R}^{-2}$ is the typical component of the Riemann tensor (see Eq. 22.23c in [9]). For the Schwarzschild metric, this entails, in terms of the frequency, the condition $\omega M \gg 1 ; M$ is the black hole mass. In the more general case of, say, the Reissner-Nordström metric, this gives $\omega r_{+} \gg 1$, where $r_{+}$is the radius of the horizon.

Also, we assume that backreaction of the photon on the metric is negligible. This implies that, in any case, its energy is much less than the ADM mass of a black hole, so in natural geometric units $\omega \ll M$. Thus we have the double inequality $r_{+}^{-1} \ll \omega \ll M$. As for the Reissner-Nordstr öm metric $M \leq r_{+} \leq 2 M$, the conditions of validity of this approximation are practically the same for the ReissnerNordström and Schwarzschild metrics. 


\section{Photon emitted at the horizon}

We see from (11) that, as the point of emission of a photon is approaching the horizon, the frequency measured at infinity becomes smaller and smaller. However, this formula does not describe what happens if the photon is emitted exactly on the horizon. Then $\omega_{0}=0$ and $l=0, f_{\mathrm{em}}=0$ from the very beginning (see also below), the photon does not reach infinity at all and moves along the leg of the horizon. Here, the original coordinates (1) are not applicable since the metric becomes degenerate on the horizon. This can be remedied with the use of standard KS coordinates or its generalization.

Let us introduce coordinates $U$ and $V$, where

$$
\begin{aligned}
U & =-\exp (-\kappa u), V=\exp (\kappa v), \\
u & =t-r^{*}, \quad v=t+r^{*} ; \\
r^{*} & =\int^{r} \frac{\mathrm{d} r}{f}
\end{aligned}
$$

is the tortoise coordinate. It is seen from (12) that

$U V=-\exp \left(2 \kappa r^{*}\right)$.

Then the metric reads

$\mathrm{d} s^{2}=F \mathrm{~d} U \mathrm{~d} V+r^{2} \mathrm{~d} \omega^{2}$.

Here,

$F=-f \frac{\mathrm{d} u}{\mathrm{~d} U} \frac{\mathrm{d} v}{\mathrm{~d} V}$.

For the transformation (12),

$F=\frac{f}{U V \kappa^{2}}$,

$F \neq 0$ on the horizon. It is clear from (1), (15) that $F=F(r)$. For example, for the Schwarzschild metric,

$F=-\frac{4 r_{+}^{3}}{r} e^{-\frac{r}{r_{+}}}$,

provided the constant of integration is chosen in (14) in such a way that $r^{*}=r+r_{+} \ln \frac{r-r_{+}}{r_{+}}$.

We consider the vicinity of the future horizon, on which $U=0$. Along this horizon, $V$ takes finite values.

Near the horizon

$$
\begin{aligned}
r^{*} & \approx \frac{1}{2 \kappa} \ln \frac{r-r_{+}}{r_{+}}+\text {const }, \\
U V & \approx \frac{\left(r-r_{+}\right)}{r_{+}} C_{0},
\end{aligned}
$$

where $C_{0}$ is a constant. In the Schwarzschild case, $C_{0}=e$. It is instructive to rewrite the equations of motion for massive particles outside the event horizon (3), (4) in terms of the KS coordinates and take the horizon limit afterwards. For an observer moving inward they read $m u^{U}=\frac{E+Z}{f} \frac{\mathrm{d} U}{\mathrm{~d} u}$,

$m u^{V}=\frac{E-Z}{f} \frac{\mathrm{d} V}{\mathrm{~d} v}$.

Taking also into account (12) and (17), we have

$m \dot{U}=-\frac{(E+Z)}{F V \kappa}$,

$m \dot{V}=\frac{(E-Z)}{U F \kappa}$.

In a similar way, we have for a photon moving in the outward direction

$k^{U}=\frac{\kappa U}{f}\left(Q-\omega_{0}\right)=\frac{Q-\omega_{0}}{F \kappa V}$,

$k^{V}=\frac{\kappa V}{f}\left(\omega_{0}+Q\right)=\frac{\omega_{0}+Q}{F U \kappa}$.

Let $\lambda$ be the affine parameter. On the future horizon $U=0$, $k^{U}=\frac{\mathrm{d} U}{\mathrm{~d} \lambda}=0=k_{V}$, which agrees with (26) if we put $\omega_{0}=0=f=Q$ in the right hand side. Thus only $k^{V}$ remains nonzero. It follows from the geodesic equations that on the horizon

$\frac{\mathrm{d} k^{V}}{\mathrm{~d} \lambda}=-\Gamma_{V V}^{V}\left(k^{V}\right)^{2}$.

Here the Christoffel symbol $\Gamma_{V V}^{V} \sim \frac{\partial F}{\partial V} \sim \frac{\partial F}{\partial r} \frac{\partial r}{\partial V} \sim f=0$ on the horizon. Therefore, $k^{V}=$ const along the horizon generator. We have from (8), (24) on the horizon

$\omega=-\frac{1}{2} F k^{V} u^{U}$,

and we have

$\omega=2 \frac{E}{V m \kappa} k^{V}$.

Let observer 1 cross the horizon at some $V=V_{1}$ and the same for observer 2 but later, at $V=V_{2}$. It follows from (12), (13) that $V_{2}>V_{1}$. Assuming that observers are identical in that they have the same values of $E$ and $m$, we obtain

$\frac{\omega_{2}}{\omega_{1}}=\frac{V_{1}}{V_{2}}<1$.

This agrees with Eq. (A16) of [3] obtained for the Schwarzschild metric by another method.

It is also instructive to check that indeed $\omega_{0}=0$. By definition, $\omega_{0}$ is a constant Killing frequency,

$\omega_{0}=-k_{\mu} \xi^{\mu}$,

where $\xi^{\mu}$ is the Killing vector. In the original coordinates (1),

$\xi^{\mu}=(1,0,0,0), \quad \xi_{\mu}=(-f, 0,0,0)$.

Passing to KS coordinates, one obtains

$\xi^{U}=-\kappa U, \xi^{V}=\kappa V$. 
Then we see from (32) that

$\omega_{0}=-F\left(k^{V} \xi^{U}+k^{U} \xi^{V}\right)=F \kappa\left(k^{V} U-k^{U} V\right)$.

On the future horizon, $k^{U}=0$ and $U=0$, so we see that indeed $\omega_{0}=0$.

Also, it is easy to check that for a photon propagating along the horizon $l=0$. Indeed, if we write down the condition $k_{\mu} k^{\mu}=0$ on the future horizon, we obtain $k_{\phi}=0$. This agrees with previous observations concerning the properties of trajectories on the horizon $[10,11]$.

\section{Generalized Lemaitre frame}

\subsection{Form of metric}

It is instructive to reformulate the redshift value in the Lemaitre-like coordinates $\rho, \tau$. In contrast to the Kruskal ones, this frame is based on free falling particles. The Lemaitre frame is well known for the Schwarzschild metric. Now, we suggest its generalization valid for the metric (1).

The general theory of transformations that make the metric of a spherically symmetric black hole regular, was developed in [12]. For our goals, it is sufficient to find a particular class of transformations that (i) makes the metric regular on the horizon, (ii) generalize the Lemaitre metric (in particular, the metric should have $g_{\tau \tau}=-1$ ). We make the transformation

$\rho=t+\int \frac{\mathrm{d} r^{*}}{\sqrt{1-f}}$,

$\tau=t+\int \mathrm{d} r^{*} \sqrt{1-f}$,

where $r^{*}$ is given by (14). Equations (36), (37) are direct generalizations of Eqs. 102.1 of [8]. Then it is easy to check that

$\mathrm{d} s^{2}=-\mathrm{d} \tau^{2}+(1-f) \mathrm{d} \rho^{2}+r^{2}(\rho, \tau)\left(\mathrm{d} \theta^{2}+\sin ^{2} \theta \mathrm{d} \phi^{2}\right)$.

On the horizon, $f=0$, the metric coefficient is regular, $g_{\rho \rho}=1$. In the particular case of the Schwarzschild metric, $f=1-\frac{r_{+}}{r}$ and we return to the standard formula for the Lemaitre metric, when $r$ is expressed in terms of $\rho$ and $\tau$. The coordinates (36), (37) are suitable for the description of a black hole including both the outer $\mathrm{R}$ region and the contracting $\mathrm{T}$ one [13]. In a similar way, one can use the expanding version that would result in a changing sign at $\tau$.

Now, we want to pay attention to some nice properties of the metric (38). The proper distance between points 1 and 2 calculated for a given $\tau$ is equal to $l=\int \mathrm{d} \rho \sqrt{1-f}$. Requiring $\mathrm{d} \tau=0$ in (37) and substituting $\mathrm{d} t$ into (36), we obtain from (14), (36) $l=r_{2}-r_{1}$.

It is also instructive to calculate the velocity. Let, say, point 1 be fixed and let us focus on the velocity of free fall $v=\frac{\mathrm{d} l}{\mathrm{~d} \tau}$ of a particle with $E=m$, where $r_{2} \equiv r$ changes depending on time. Then it is easy to find from (4), (39) that

$v=-\sqrt{1-f}$

Taking the derivative once more, we obtain $\frac{\mathrm{d} v}{\mathrm{~d} r}=\frac{1}{2 \sqrt{1-f}} \frac{\mathrm{d} f}{\mathrm{~d} r}$. On the horizon, this gives us

$$
\left(\frac{\mathrm{d} v}{\mathrm{~d} r}\right)_{H}=\kappa,
$$

where we took into account that for our metric the surface gravity $\kappa=\frac{1}{2}\left(\frac{\mathrm{d} f}{\mathrm{~d} r}\right)_{H}$. The subscript " $\mathrm{H}$ " means that the corresponding quantity is calculated on the horizon. Equation (41) will be used below. It is worth noting that for the extremal horizon $(\kappa=0)$ we have also $\left(\frac{\mathrm{d} v}{\mathrm{~d} r}\right)_{H}=0$.

\subsection{Redshift: from Kruskal coordinates to Lemaitre ones}

The above frame is especially useful for the presentation of the redshift (31). On the horizon, $f=0$. Then, in its vicinity, we obtain from (13), (36), (37) that on the horizon

$v=\tau+C_{1}=\rho+C_{2}$,

where $C_{1,2}$ are constants. As a result, we obtain from (31)

$\frac{\omega_{2}}{\omega_{1}}=\exp \left(\kappa\left(\tau_{1}-\tau_{2}\right)\right)=\exp \left(\kappa\left(\rho_{1}-\rho_{2}\right)\right)$.

Thus the Lemaitre frame allows us to present the resulting redshift along the horizon in a simple and intuitively clear picture - the redshift grows (and, consequently, the emitter looks dimmer) exponentially with respect to Lemaitre time that passes from emitting to observation.

In the last paragraph of Sect. 2, we listed the general condition for the geometrical optic to be valid. Now, we can express it in another way. Since a physical wave packet has a finite length, parts of it will move away from the black hole horizon even if its center is located exactly on the horizon. Since the equations of light geodesics in the generalized Lemaitre frame reads $\mathrm{d} r / \mathrm{d} \tau=1-\sqrt{1-f}$ for outward propagation, the Lemaitre time needed to leave the vicinity of the horizon $r=r_{+}$diverges as $\left|\ln \left(r / r_{+}-1\right)\right|$. Suppose, the emitter radiates light with the wavelength $\lambda$. Since in any case the wave packets cannot be smaller than $\lambda$, we can roughly estimate initial scale as $r-r_{+} \sim \lambda$. Then we find that after the Lemaitre time $\tau / r_{+} \sim \ln r_{+} / \lambda \sim \ln \omega_{0} r_{+}$the wave packet will reach the scale of black hole horizon, the geometric optic approximation fails and, in particular, Eq. (43) evidently breaks down. 


\section{Photon emitted at the inner horizon}

Let us consider a situation similar to that considered above. An observer moves beyond the event horizon $r_{+}$and approaches the inner horizon $r_{-}<r_{+}$. When he crosses it, he emits a photon. Another observer who also crosses the inner horizon later, receives this very photon. What can be said about its frequency?

\subsection{The coordinates and metric}

The metric between the outer and inner horizons represents so-called $T$ region [13]. For definiteness, we consider the $T^{-}$ region that corresponds to a black hole, but similar formulas are valid for the $T^{+}$regions (white holes). Now, the metric can be formally obtained from (1) if one takes into account that for $r_{-}<r<r_{+}$the metric function $f<0$, so spacelike and timelike coordinates mutually interchange. We can write $f=-g$,

$y=t, \quad T=-r$.

Then the metric can be rewritten in the form

$\mathrm{d} s^{2}=-\frac{\mathrm{d} T^{2}}{g}+g \mathrm{~d} y^{2}+T^{2} \mathrm{~d} \omega^{2}$.

The equations of motion for a massive particle have the form

$m \dot{y}=\frac{P}{g}$,

$m \dot{T}=z \equiv \sqrt{P^{2}+m^{2} g}$,

where $P=m u_{y}$ is the conserved momentum.

Now, the KS transformation somewhat changes and reads

$U=\exp \left(-\kappa_{-} u\right)$

$V=\exp \left(\kappa_{-} v\right)$

where $r^{*}$ is given by the formula

$r^{*}=\int \frac{\mathrm{d} r}{g}$.

The metric takes the form (16) with

$F=-\frac{g}{U V \kappa_{-}^{2}}$,

where $\kappa_{-}$is the surface gravity associated with the inner horizon and $F \neq 0$ is finite there.

Repeating the calculations step by step, we arrive at the same formula (31),

$\frac{\omega_{2}}{\omega_{1}}=\frac{V_{1}}{V_{2}}$.

Now, we would like to pay attention to the fact that, according to (12), $V$ is a monotonically increasing function of $v$. It is seen from (13), (14) that, for a fixed $u, \frac{\partial v}{\partial r}>0$. However, it is seen from (44) that event 2, which takes place after 1, has $r_{2}<r_{1}$. As a result, $v_{2}<v_{1}$ and $V_{2}<V_{1}$. Therefore, $\omega_{2}>\omega_{1}$ and now we have a blueshift. Thus this is related to the fact that $r$ and $t$ coordinates change their character in the region under discussion.

The results (43) and (52) can be united in one formula,

$$
\frac{\omega_{2}}{\omega_{1}}=\exp \left[\left(\frac{\mathrm{d} v}{\mathrm{~d} r}\right)_{H}\left(\rho_{1}-\rho_{2}\right)\right]=\exp \left[\left(\frac{\mathrm{d} v}{\mathrm{~d} r}\right)_{H}\left(\tau_{1}-\tau_{2}\right)\right],
$$

where $\tau_{2}>\tau_{1}, \rho_{2}>\rho_{1}$. For the outer horizon we can use Eq. (41), which gives us (43) and we have a redshift. For the inner horizon, the counterpart of (41) gives us $\left(\frac{\mathrm{d} v}{\mathrm{~d} r}\right)_{H}=-\kappa_{-}$, where now $\kappa_{-}=\frac{1}{2}\left|\frac{\mathrm{d} f}{\mathrm{~d} r}\right|_{H}$ is the surface gravity of the inner horizon (where $\left(\frac{\mathrm{d} f}{\mathrm{~d} r}\right)_{H}<0$ ). As a result, we obtain here a blueshift.

In a similar way, the procedure under discussion gives the same result when an observer crosses the event horizon of a white hole moving outward from the $T^{+}$to the $R$ region. Then he will find all photons propagating along this horizon to be blueshifted. In particular, this holds for the Schwarzschild metric. Analogously, an observer entering $T^{+}$region from the inner $R$ one (say, like in the ReissnerNordström metric) will see a redshift at the inner horizon. In other words, in both situations (either black or white hole) an observer crossing a horizon from the $T$ to $R$ region will see a blueshift, while from the $R$ to $T$ region he will see a redshift.

\subsection{Relation to other effects}

In the previous subsection we have shown that the blueshift at the inner horizon (and, consequently, the energy absorbed by the observer) grows exponentially with the Lemaitre time between the moments of emission and observation. Here we compare this interesting effect with others known in the literature.

If two particles collide, their energy $E_{\text {c.m. }}$ in the center of mass frame can be defined on the point of collision according to

$E_{\mathrm{c} . \mathrm{m} .}^{2}=-P_{\mu} P^{\mu}$,

$P^{\mu}=p_{1}^{\mu}+p_{2}^{\mu}$ being the total momentum of two particles. If particle 1 is massive and particle 2 is massless, $p_{1}^{\mu}=m u^{\mu}$ and $p_{2}^{\mu}=k^{\mu}$, where we put the Planck constant to unity. As a result,

$E_{\text {c.m. }}^{2}=m^{2}+2 m \omega$.

In the example under discussion, if $V_{1}=O(1)$ and $V_{2} \rightarrow$ 0 , the frequency $\omega_{2} \rightarrow \infty$ according to (52). Then $E_{\text {c.m. }} \rightarrow$ 
$\infty$ as well and we encounter a counterpart of the BSW effect near the inner horizon. But $V=0$ on the future horizon $U=0$ is nothing else than the bifurcation point [5] (see also below for more details). Thus the present results for the blueshift agree with the previous ones in the limit when the bifurcation point is reached.

There is also another issue to which we can compare the present consideration. As is well known, near the inner (Cauchy) horizon an instability develops inside black holes. This happens when a decaying flux of radiation coming from infinity crosses the event horizon and concentrates near the inner one-see, e.g. Chapter 14.3.1 in [6]. (For a modern review of the subject see [7].) However, now we consider radiation which is not coming from infinity but is emitted by an observer who crosses the inner horizon. The resulting energy flux from an emitter at the inner horizon appears to be finite, though it is not restricted from above if $V \rightarrow 0$.

Thus as far as the radiation near the inner horizon is concerned, we have three situations: (i) the analog of the BSW effect (relevant near the bifurcation point), (ii) blueshift of a photon in the situation under discussion (relevant near any point of the inner horizon, the blueshift is in general finite), (iii) the instability of the inner horizon (infinite blueshift due to concentration of radiation along the horizon). Cases (i) and (ii) are closely related in the sense that in the limit when the point where a photon is absorbed approaches the bifurcation point, one obtains (i) from (ii). Meanwhile, in case (iii) the effect is unbounded and this points to a potential pathology connected with the nature of the inner horizon.

\section{Special case: emission at the bifurcation point}

In Sect. 5, we discussed briefly spacetimes that contain $T^{+}$ regions (white holes). Then the intersection between the future and past horizons forms the so-called bifurcation point (sphere, if the angle variables are taken into account), where it is possible to pass from the white hole region to the black one. White holes and bifurcation points do not arise in the situation when a black hole is formed due to gravitational collapse and in this sense they are not feasible astrophysically. However, they are inevitably present in the full picture of an eternal black-white hole. Therefore, we consider such objects for theoretical reasons and for completeness. In particular, in Sect. 5, we saw that accounting for the bifurcation point arises naturally in the connection between our problem and the BSW effect. In doing so, it is a receiver that passes near the bifurcation point.

In the present section, we consider another case, when it is an emitter that passes through this point at the moment of radiation. Consideration of the frequency shift when a photon emitted from the bifurcation point is a separate case that does not follow directly from the previous formulas.
For the Reissner-Nordström-de Sitter metric, such a problem was considered in Sect. IV b of [1]. It follows from the corresponding results that different cases are possible here: $\omega_{2}<\omega_{1}, \omega_{2}=\omega_{1}, \omega_{2}>\omega_{1}$. On the first glance, this disagrees with our results described above since we obtained either a redshift (for the event horizon of black hole or inner horizon of a white hole) or a blueshift (for the inner one in a black hole or event horizon of a white hole). Fortunately, this contradiction is illusory. Now, we will explain how one can obtain the results for the bifurcation point from ours. To this end, we compare (i) the generic situation and (ii) that with crossing the bifurcation point and trace how (ii) arises from (i) within the limiting transition.

For our purposes, it is sufficient to discuss the simplest metric that possesses the bifurcation point, so we can imply it to be, say, the Schwarzschild one. We assume that emitter 1 moves from the inner expanding $T^{+}$region (i.e. white hole) [13], crosses the past horizon and enters the R region. Afterwards, it crosses the event horizon falling into a black hole. Let, as before, the emitter and receiver have equal masses $m_{1}=m_{2}=m$. However, now we cannot put $E_{1}=E_{2}$. This is because a particle with $E=m$ would escape to infinity instead of falling into a black hole. We remind the reader that, up to now, in all our considerations an emitter and an observer are set to be at rest in infinity. However, the most general case can easily be obtained by adding corresponding Lorentz boosts. In the present subsection we meet the situation where this procedure is needed.

Therefore, we must use a more general formula based on (30),

$\frac{\omega_{2}}{\omega_{1}}=\frac{E_{2}}{E_{1}} \frac{V_{1}}{V_{2}}$.

The first factor can be interpreted as a Lorentz boost responsible for the Doppler effect. For $E_{1}=E_{2}$ we return to the case considered by us above but now the first factor is not equal to 1 and plays now a crucial role.

If, by assumption, particle 1 falls into a black hole, this means that it must bounce from the potential barrier in the turning point $r=r_{0}$. According to the equation of motion (4), this means that

$E=m \sqrt{f\left(r_{0}\right)}$.

If $r_{0} \rightarrow r_{+}, f\left(r_{0}\right) \rightarrow 0$, so $E \rightarrow 0$ as well. More precisely, it is seen from (2), (21) that

$E \sim \sqrt{r_{0}-r_{+}} \sim \sqrt{|U| V}$.

As a result,

$\frac{\omega_{2}}{\omega_{1}} \sim \frac{\alpha E_{2}}{V_{2}}, \quad \alpha \equiv \sqrt{\frac{|V|_{1}}{U_{1}}}$.

In the limit when the trajectory of particle 1 passes closer and closer to the bifurcation point $U=0=V, \alpha$ remains 
finite. Using the equations of motion in the $\mathrm{T}$ region (see the previous section), it is easy to show that in the limit $V \rightarrow 0$, $U \rightarrow 0$, the component of the velocity $u^{U}$ contains just this factor $\alpha$.

Thus depending on the relation between $\alpha$ and $V_{2}$ one can obtain any result for $\omega_{2}$ (redshift, blueshift, the absence of the frequency shift). In this sense, the general formula (56) reproduces both the "standard" fall of the emitter in a black hole and the behavior of the emitter that passes through the bifurcation point.

\section{Extremal horizon}

Let an observer cross the (ultra) extremal horizon $r_{+}$. By definition, this means that near it the metric function is

$f \sim\left(r-r_{+}\right)^{n}$

where $n=2$ in the extremal case and $n=3,4 \ldots$ in the ultraextremal one. The difference from the nonextremal case consists in a different nature of the transformation making the metric regular. Let the two-dimensional part of the metric have the same form as in (1). The subsequent procedure is well known—see, e.g., [14,15] (Sect. 3.5.1). We use the same coordinates $u, v$ and want to find appropriate coordinates $U$, V,

$V=V(v), \quad U=U(u)$.

Now, we are interested in the situation with emission of a photon exactly along the horizon. Near the horizon it follows for the tortoise coordinate (14) that

$$
\begin{aligned}
r-r_{+} & \sim\left|r^{*}\right|^{\frac{1}{1-n}}, \\
f & \sim\left|r^{*}\right|^{\frac{n}{1-n}} .
\end{aligned}
$$

We consider the metric near the future horizon where $v$ is finite, $r^{*} \rightarrow-\infty, u=v-2 r^{*} \rightarrow+\infty$. We have

$f \sim u^{\frac{n}{1-n}}$.

We try a transformation that behaves like

$$
U \sim u^{-\frac{1}{n-1}}
$$

so that $U \rightarrow 0$. Then it is easy to check that the metric has the form (16) where $F \neq 0$ is finite on the horizon. To find the frequency, we must use the expression for $u^{U}$ (22) in which now (65) is valid, so $\frac{d U}{d u} \sim u^{\frac{n}{1-n}}$. It is seen from (64) that $u^{U} \rightarrow$ const on the horizon and it does not contain $V$. Taking into account that $k^{V}$ is a constant along the horizon generator as before, we come to the conclusion that $V$ drops out and $\frac{\omega_{2}}{\omega_{1}}=$ const. We see that in the horizon limit the quantity $V$ does not enter the frequency. In this sense, $\frac{\omega_{2}}{\omega_{1}}$ does not change along the horizon, so redshift or blueshift is absent.
In a sense, this is quite natural. Indeed, the extremal horizon is the double one. The inner and outer horizons merge. But for an inner horizon we had a blueshift, for the outer one we had a redshift. Together, they mutually cancel and produce no effect.

The absence of the redshift or blueshift formally agrees with (43) if one puts $\kappa=0$ there. However, for (ultra)extremal black holes the Kruskal-like transformation looks very different, so we could not use Eq. (43) directly. Therefore, it was not obvious in advance whether or not the redshift for the extremal horizon can be obtained as the extremal limit of a nonextremal one. Now, we see that this is the case.

\section{Summary}

Thus we showed that for emission along the outer horizon redshift occurs and we derived a simple formula that generalized the one previously found in the literature. We also showed that along the inner horizon blueshift occurs and found its relation with the BSW effect. We also showed how the previously known results for the emission at the bifurcation point are reproduced from a general formula and lead to a diversity of situations (redshift, blueshift or the absence of frequency shift). For (ultra)extremal horizons the effect is absent.

These observations have a quite general character in agreement with the universality of black hole physics. We also generalized the Lemaitre frame and in this frame derived a simple and instructive formula for a redshift along the horizon in terms of the Lemaitre time and the surface gravity.

Acknowledgements The work was supported by the Russian Government Program of Competitive Growth of Kazan Federal University.

Open Access This article is distributed under the terms of the Creative Commons Attribution 4.0 International License (http://creativecomm ons.org/licenses/by/4.0/), which permits unrestricted use, distribution, and reproduction in any medium, provided you give appropriate credit to the original author(s) and the source, provide a link to the Creative Commons license, and indicate if changes were made. Funded by SCOAP ${ }^{3}$.

\section{References}

1. K. Lake, Reissner-NorBstrom-de Sitter metric, the third law, and cosmic censorship. Phys. Rev. D 19, 421 (1979)

2. A.T. Augousti, M. Gawełczyk, A. Siwek, A. Radosz, Touching ghosts: observing free fall from an infalling frame of reference into a Schwarzschild black hole. Eur. J. Phys. 33, 1-11 (2012)

3. K. Kassner, Why ghosts don't touch: a tale of two adventurers falling one after another into a black hole. Eur. J. Phys. 38, 015605 (2017). arXiv: 1608.07511

4. M. Bañados, J. Silk, S.M. West, Kerr Black holes as particle accelerators to arbitrarily high energy. Phys. Rev. Lett. 103, 111102 (2009). arXiv:0909.0169 
5. O.B. Zaslavskii, Acceleration of particles near the inner black hole horizon. Phys. Rev. D 85, 024029 (2012). arXiv:1110.5838

6. V.P. Frolov, I.D. Novikov, Physics of black holes. Basic Concepts and New Developments (Kluwer Academic Publishers, Dordrecht, 1998)

7. A.J.S. Hamilton, A.P.P. Avelino, Phys. Rep. 495, 1 (2010)

8. L.D. Landau, E.M. Liefshitz, The Classical Theory of Fields (Pergamon Press, New York, 1971)

9. C.W. Misner, K.S. Thorne, J.A. Wheeler, Gravitation (W. H. Freeman and company, San Francisco, 1973)

10. T. Harada, M. Kimura, Collision of an innermost stable circular orbit particle around a Kerr black hole. Phys. Rev. D 83, 024002 (2011). arXiv:1010.0962
11. O.B. Zaslavskii, Near-horizon circular orbits and extremal limit for dirty rotating black holes. Phys. Rev. D 92, 044017 (2015). arXiv: 1506.00148

12. P.I. Fomin, Coordinate transformation that eliminate singularities on the gravitational radius in the Schwarzschild metric. Sov. Phys. JETP 27, 483 (1968)

13. I.D. Novikov, Commun. Sternberg Astron. Inst. 132(3), 132 (1964)

14. S. Liberati, T. Rothman, S. Sonego, Nonthermal nature of incipient extremal black holes. Phys.Rev. D 62, 024005 (2000). arXiv:gr-qc/0002019

15. K.A. Bronnikov, S.G. Rubin, Black holes, Cosmology and Extra dimensions (World Scientific, 2013) 